\title{
Lapatinib, a TKI Dual Inhibitor of Her1 and Her2 Receptors: Review of the Literature*
}

\author{
Vito Lorusso $^{1 \#}$, Ilaria Marech ${ }^{1}$, Marianna Giampaglia ${ }^{2}$, Antonio Gnoni ${ }^{2}$, Andrea Tinelli ${ }^{3}$ \\ ${ }^{1}$ National Cancer Research Center, Medical Oncology Unit, Istituto Tumori “Giovanni Paolo II”, Bari, Italy; ${ }^{2}$ Medical Oncology Unit, \\ Vito Fazzi Hospital, Lecce, Italy; ${ }^{3}$ Gynecology and Obstetrics Department, Vito Fazzi Hospital, Lecce, Italy. \\ Email: \#vitolorusso@me.com
}

Received August $10^{\text {th }}, 2012$; revised September 11 ${ }^{\text {th }}, 2012$; accepted September $23^{\text {rd }}, 2012$

\begin{abstract}
Lapatinib ditosylate $\left(\right.$ Tyverb $\left.^{\circledR}\right)$ is a potent and selective oral dual receptor tyrosine kinase inhibitor (TKI), preventing autophosphorylation of epidermal growth factor receptor (EGFR/ErbB1) and human epidermal growth factor receptor 2 (HER2/ErbB2) intracellular domain. This interference blocks the Ras/Raf MAPKs and PI3K/Akt pathways, that lead to uncontrolled cellular proliferation and survival. After the demonstration of its effectiveness and safety in HER2-overexpressed breast cancer, in 2007 the US Food and Drug Administration (FDA) approved this molecule in combination with capecitabine, in patients with locally advanced or metastatic disease, that progressed after previous treatment with anthracyclines, taxanes and trastuzumab. In 2010, Lapatinib received approval for the treatment of postmenopausal women with hormone receptor positive metastatic breast cancer in combination with letrozole. The most common adverse events (AE) are: anorexia, insomnia, diarrhea and skin rash and mild cardiovascular toxicity. This paper reviews the most important studies on Lapatinib in advanced breast cancer. However, promising results were recently reported on this drug, also in adjuvant setting and in combination with other target drugs, which warrant further investigation for the future.
\end{abstract}

Keywords: Lapatinib; Tyverb ${ }^{\circledR}$; HER2; Breast Cancer; TKI

\section{Introduction}

Recently, the field of cancer therapy has witnessed the emergence of multiple targeted agents able to inhibit specific key molecules and crucial pathways for tumor growth and progression. Among the mechanism of pathogenesis of breast cancer (BC) there is, in many cases, an altered regulation of the HER-mediated signaling network, through the amplification of the HER2 gene, which results in HER2 protein over-expression. This alteration is present in $20 \%-25 \%$ of human $\mathrm{BC}$ and it's an adverse prognostic factor, associated with aggressive histopathological parameters, decreased disease-free survival and overall survival (OS) [1].

Recent studies have shown that EGFR-specific inhibitors (monoclonal antibodies and small molecules) can reduce HER2-signaling and growth of BC cells, expressing high levels of HER2.

Trastuzumab, a humanized monoclonal antibody, targeting the HER2 extracellular domain, represented a real clinical breakthrough in the treatment of HER2-positive

\footnotetext{
"Disclosure: The authors have indicated no financial relationships with companies whose products are mentioned in this article.

"Corresponding author.
}

$\mathrm{BC}$, showing synergism with chemotherapy in metastatic as well as in adjuvant or neo-adjuvant setting. In addition, it has been postulated that trastuzumab may be of benefit beyond disease progression. However, this point remains controversial [2].

Lapatinib, a small-molecule, reversible inhibitor of both ErbB1 and HER2, has mechanisms of action distinct from trastuzumab. This small molecule works intra-cellularly, reversibly binding to the cytoplasmic ATP-binding site of the kinase and blocking receptor phosphorylation and activation. This interaction prevents the signal transduction of both Ras/Raf mitogen-activated protein kinases (MAPKs) and PI3K (phosphatidylinositol-3kinase)/Akt pathways, leading to an increase in apoptosis and decrease cellular proliferation [3] (Figure 1).

In preclinical studies, lapatinib demonstrated to be not cross-resistant with trastuzumab, and clinical evidence suggests that its activity does not depend on the PTEN, p95 HER2 (a truncated version of HER2 receptor) [4], IGF1R or PI3K mutation status [5,6]. Several phase II and III trials have demonstrated a substantial anti-tumor activity of lapatinib against HER2-overexpressing metastatic BC (MBC). Moreover, the drug appeared to be generally well tolerated, with diarrhea, skin rash, fatigue 


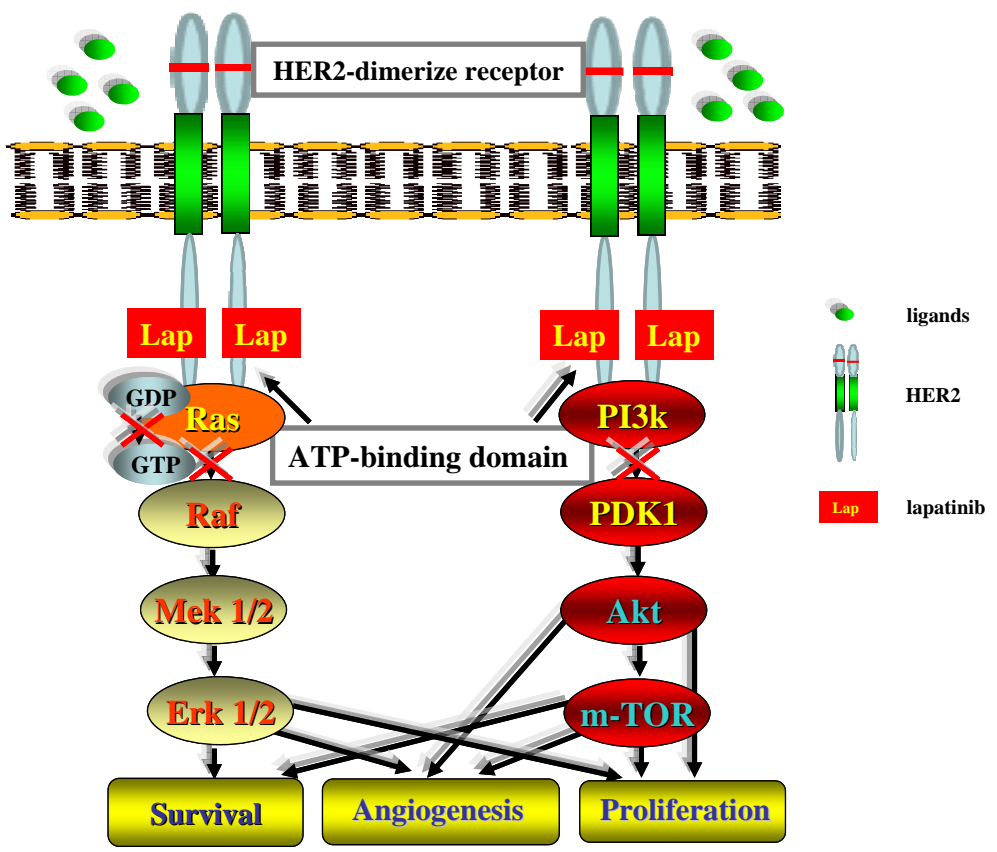

Figure 1. Lapatinib binds to the tyrosine kinase (TK) domain, competitively blocking ATP binding, thus inhibiting the downstream cascade of events (reprinted with permission from Giampaglia M. et al.: "Lapatinib in breast cancer: clinical experiences and future perspectives”. Cancer Treatment Reviews 2010 Nov; 36 Suppl 3: S72-9).

and nausea being its main toxicities. Compared to trastuzumab, lapatinib is also less cardiotoxic. A retrospective review of cardiac safety on 3689 patients, who had received lapatinib, found an overall decrease of left ventricular ejection fraction (LVEF) (defined as $20 \%$ fall relative to baseline and below the institution's lower normal limit) in $1.6 \%$ of patients, with only $0.2 \%$ symptomatic [7].

\section{Lapatinib in Combination with Endocrine Agents}

Despite recent progresses in the treatment of hormone receptor (HR)-positive metastatic $\mathrm{BC}$, the resistance to endocrine therapies limits their long-term success. Crosstalk between pathways involving the epidermal growth factor family of receptors (ErbB1 and HER2) and the estrogen receptor (ER) is involved in resistance to endocrine therapy [8-10]. Moreover, targeted agents have been used in preclinical models to enhance the efficacy of either tamoxifen or estrogen deprivation [1-13]. This constituted the rationale for using targeted agents against EGFR pathways in combination with endocrine manipulation to overcome endocrine resistance.

A randomized trial (TAnDEM trial: Trastuzumab and Anastrozole Directed Against ER-Positive HER2-Positive Mammary Carcinoma) in dual HR-positive and HER2-positive MBC, reported that trastuzumab combined with the aromatase inhibitor (AI) anastrozole doubled the median progression-free survival (PFS) com- pared to anastrozole alone (from 2.4 to 4.8 months) [14] A phase III randomized trial in post-menopausal HRpositive MBC $(n=1286)$ compared the combination of letrozole with lapatinib $(\mathrm{n}=642)$ vs letrozole alone $(\mathrm{n}=$ 644) [15]. Prior neoadjuvant/adjuvant anti-estrogen therapy was allowed, as were adjuvant aromatase inhibitors and trastuzumab if discontinued $>12$ months prior to trial entry. In women with HR-positive HER2-positive disease $(\mathrm{n}=219)$, after a median follow up of 1.8 years, the combination of letrozole-lapatinib was superior to letrozole alone in terms of median PFS (8.2 vs 3.0 months, HR $=0.71,95 \%$ CI 0.53 to $0.96, \mathrm{p}=0.019$ ). Consistent with these findings, a superior clinical benefit rate $(\mathrm{CBR})$ of $48 \%$ vs $29 \%(\mathrm{p}=0.003)$ was observed. With less than $50 \%$ of overall survival (OS) events yet recorded, the median OS in the HER2-positive population was 32.3 months in letrozole placebo arm compared with 33.3 months in the combination arm $(\mathrm{HR}=0.74$; $95 \%$ CI, 0.5 to $1.1 ; p=0.113)$. The most common adverse reactions were: diarrhea, rash, nausea, arthralgia and fatigue (majority were grade 1 or 2), with a higher incidence in the combination arm for diarrhea and rash. Treatment-related LVEF decline and elevation of liver function were infrequent.

\section{Lapatinib in the Neoadjuvant Setting}

Neoadjuvant (primary systemic) chemotherapy is currently used for operable, early-stage $\mathrm{BC}$ with several advantages [16], as increase in the rate of breast-con- 
serving surgery or in vivo assessment of tumor response. The addition of trastuzumab to neoadjuvant chemotherapy has shown to significantly increase pCR (pathological Complete Remission), breast sparing surgery and event-free survival in women with operable, HER2positive disease $[17,18]$. The phase III randomized study NeoALTTO [19] (Neoadjuvant Lapatinib and/or Trastuzumab Treatment Optimization) was designed to compare the efficacy of neoadjuvant lapatinib, trastuzumab or their combination, in association with paclitaxel. Patients were randomized to receive either lapatinib 1500 $\mathrm{mg}$ daily; trastuzumab $4 \mathrm{mg} / \mathrm{kg}$ intravenous (IV) load followed by $2 \mathrm{mg} / \mathrm{kg}$ IV weekly, or lapatinib $1000 \mathrm{mg}$ daily with trastuzumab $4 \mathrm{mg} / \mathrm{kg}$ IV load followed by 2 $\mathrm{mg} / \mathrm{kg}$ IV weekly for a total of 6 weeks [20]. After this "biological window", all patients continued on the targeted therapy plus weekly paclitaxel $80 \mathrm{mg} / \mathrm{m}^{2}$ for a further 12 weeks period, up to definitive surgery. After surgery, patients received 3 courses of adjuvant chemotherapy with 5-fluorouracil, epirubicin, and cyclophosphamide (FEC), followed by the identical targeted therapy given as neoadjuvant for further 34 weeks (i.e. trastuzumab every 3 weeks, lapatinib or the combination). Patients received study treatments for 1 year (18 weeks pre-surgery, and 34 weeks post-surgery). A total number of 455 women with HER2-positive breast cancer BC in about 130 sites in approximately 30 countries have been recruited. The primary study endpoint was the pCR rate at the time of surgery (time frame: 20 - 22 weeks) as defined by National Surgical Adjuvant Breast and Bowel Project (NSABP) criteria. The pCR rate was $51.3 \%$ for patients receiving a combination of trastuzumab and lapatinib, significantly higher than $\mathrm{pCR}$ rate observed in the trastuzumab alone arm $(29.5 \% ; p=0.0001)$ or in the lapatinib alone arm $(24.7 \%, \mathrm{p}=$ not significant vs. trastuzumab alone). With regard to safety of lapatinib treated patients, although no major cardiac dysfunction was reported, the study demonstrated substantially higher toxicity in both lapatinib-containing arms compared with trastuzumab alone (particularly diarrhoea, hepatic AEs, neutropenia and skin disorders) [21].

The CHERLOB (Preoperative chemotherapy plus trastuzumab, lapatinib or both) [22], is a phase IIb randomized 3 arm trial, in which patients with HER2-positive primary breast cancer stage II-IIIA (tumor size $>2$ $\mathrm{cm}$ ) were randomized to receive chemotherapy with weekly paclitaxel $80 \mathrm{mg} / \mathrm{m}^{2}$ for 12 weeks followed by 4 courses of FEC (5-fluorouracil [5-FU] $600 \mathrm{mg} / \mathrm{m}^{2}$, epirubicin $75 \mathrm{mg} / \mathrm{m}^{2}$, cyclophosphamide $600 \mathrm{mg} / \mathrm{m}^{2}$ ) administered every 3 weeks plus trastuzumab $4 \mathrm{mg} / \mathrm{kg}$ loading dose followed by $2 \mathrm{mg} / \mathrm{kg}$ weekly for 26 weeks ( $\mathrm{arm} \mathrm{A}$ ); or to receive the same chemotherapy regimen plus lapatinib $1500 \mathrm{mg}$ orally daily for the whole duration of chemotherapy (arm B); or to receive the same chemo- therapy regimen plus trastuzumab $2 \mathrm{mg} / \mathrm{kg}$ weekly (first loading dose $4 \mathrm{mg} / \mathrm{kg}$ ) plus lapatinib $1000 \mathrm{mg}$ orally daily for the whole treatment period (arm C). Surgery was planned within 2 weeks after the last trastuzumab/ lapatinib dose. The primary endpoint of this study was the percentage of pCR, defined as complete disappearance of invasive tumor in breast and axillary lymph nodes. Secondary aims were as follows: the percentage of clinical objective responses in the breast, the percentage of conservative surgery, the safety profile of these combinations, the time to treatment failure from the beginning of the primary therapy, the percentage of inhibition of intermediate and final biomarkers of the proliferative and the apoptosis pathways induced by the different combinations and the correlation between gene expression at diagnosis and pathologic response. One hundred and twenty one patients (pts) have been randomized until November 2010. Eighty pts have completed surgery and they are evaluable for response: 50 pts $(62.5 \%)$ achieved breast conservation (BCS - breast conserving surgery). A conversion from mastectomy to BCS was observed in 23/44 pts, initially considered candidates for mastectomy: the conversion rate was $52 \%$ (63\% in arm A, 53\% in arm $\mathrm{B}$ and $62 \%$ in arm $\mathrm{C})$. The pCR rate was $36.2 \%(28 \%$ in arm $\mathrm{A}, 32 \%$ in arm $\mathrm{B}$, and $48 \%$ in arm C). By using a $30 \%$ cut-off for $\mathrm{p} 95$ positivity, in a preliminary analysis of 48 cases, $57 \%$ resulted as p95 positive. In this preliminary analysis, the pCR rate in 15 trastuzumab treated pts was $86 \%$ in p95-negative and $13 \%$ in p95 positive cases, respectively. Mean LVEF range was $62 \%$ (52\% $77 \%)$ at baseline, $61 \%(44 \%-78 \%)$ after $12-13$ weeks and $61 \%(44 \%-74 \%)$ at the end of the therapy, respectively. No patient had symptomatic cardiac events.

In TBCRC 006 [23] a multicenter phase II study, in HER2-positive breast cancer, patients received daily Lapatinib (1000 mg for 12 weeks) and weekly Trastuzumab (4 mg/kg loading then $2 \mathrm{mg} / \mathrm{kg}$ ). The patients ER positive also received letrozole (plus goserelin if premenopausal), to block ER/HER cross-talk. Adverse events (AEs) were negligible. Overall pathologic response rate (pRR) was 53\% (ER+: 56\%, ER-: 48\%). The overall pCR rate was $28 \%$ (ER+: $21 \%$, ER-: $40 \%$ ).

The aim of the NSABP trial B-41 [24] was to determine the effect of substituting lapatinib (L) for trastuzumab (T) in combination with weekly paclitaxel (WP) following doxorubicin/cyclophosphamide (AC), as well as adding $\mathrm{L}$ to $\mathrm{T}$ with WP following $\mathrm{AC}$ on pathologic complete response (pCR) rates in neoadjuvant chemotherapy [24]. Results were available from 519 of 529 patients. The pCR percentage was $52.5 \%$ for $\mathrm{AC} \rightarrow \mathrm{WP}$ $+\mathrm{T}, 53.2 \%(\mathrm{p}=0.9)$ for $\mathrm{AC} \rightarrow \mathrm{WP}+\mathrm{L}$, and $62 \%(\mathrm{p}=$ $0.075)$ for $\mathrm{AC} \rightarrow \mathrm{WP}+\mathrm{TL}$, respectively. Grade $3 / 4$ toxicities included diarrhea in 2\%,20\%,27\% ( $<<0.001)$, and symptomatic grade $3 / 4$ left ventricular systolic dys- 
function in $4 \%, 4 \%$, and $2 \%(\mathrm{p}=0.49)$. The authors concluded that substitution of lapatinib for trastuzumab in combination with the chemotherapy program, employed in this study, resulted in similar high percentages of $\mathrm{pCR}$. Combined HER2-targeted therapy produced a numerically higher pCR percentage than single agent HER2directed therapy, but the difference was not statistically significant.

\section{Lapatinib in the Adjuvant Setting}

The role of lapatinib, dual tyrosine kinase (TK) inhibitor, in the adjuvant setting in HER2-positive breast cancer is currently being investigated. This small molecule seems to be the ideal candidate in the treatment of HER2-positive breast cancer for several reasons: 1) the oral administration; 2) the mechanism of action different from trastuzumab; 3) the demonstrated activity in patients with a truncated version of HER2 [25]; 4) the demonstrated efficacy in trastuzumab-pretreated and resistant patients; 5 ) the promising results in patients with central nervous system (CNS) disease [26].

The phase III ongoing study ALTTO (Adjuvant Lapatinib and/or Trastuzumab Treatment Optimization) is a four-arm randomized trial designed to compare lapatinib (arm A), trastuzumab (arm B), trastuzumab followed by lapatinib (arm C) or concurrent treatment with both agents (arm D) for early stage HER2-positive BC [27]. The study completed enrollment, but the results have not been published yet. However, on September 9th 2011, the leadership of ALTTO announced that it will discontinue Arm A of the trial (Lapatinib alone). The Independent Data Monitoring Committee review of efficacy in the trial was triggered after a pre-specified number of events was reached, as outlined in the study's protocol. The committee has indicated that the lapatinib alone arm is unlikely to meet the pre-specified criteria to demonstrate non-inferiority to trastuzumab alone with respect to disease-free survival. Consequent to this finding, patients assigned to the lapatinib alone arm of the trial will discontinue lapatinib and discuss treatment options with their study physician. This study is fully recruited and the remaining three arms of the trial will continue as planned.

The randomized phase III study TEACH [28] (Tyverb Evaluation After Chemotherapy) was designed to test lapatinib efficacy in patients with HER2-positive early breast cancer. Participants were randomized to receive lapatinib $1500 \mathrm{mg}$ or matching placebo orally administered once daily. The treatment was continued for a maximum of 12 months or until disease recurrence or unacceptable toxicity. Disease-free survival (DFS) was considered as the primary efficacy endpoint. The results were presented at the 2011 San Antonio Breast Cancer Symposium. After a median follow up of 4 years, DFS events occurred in $13 \%$ of patients in the lapatinib arm and $17 \%$ of patients in the placebo arm of the trial (Hazard Ratio $=0.8395 \%$; CI: 0.70 to 1.00 ; stratified logrank 2 -sided $\mathrm{p}=0.053$ ). Therefore, although an improvement in disease-free survival in favor of lapatinib was observed, this result did not meet the pre-specified criteria for statistical significance.

\section{Lapatinib in the Metastatic Setting}

Lapatinib was approved by the FDA on March 13th 2007, based on the interim results of a randomized, open-label, Phase III trial which enrolled 324 women locally advanced breast cancer or MBC (HER2-positive) progressing after treatment with regimens containing anthracycline, taxane, and trastuzumab [29]. Patients were assigned to receive either lapatinib in combination with capecitabine or single-agent capecitabine.

Both treatments were administered until tumor progression or unacceptable toxicity. The results showed that the addition of lapatinib to capecitabine was associated with $51 \%$ reduction in the risk of disease progression ( $\mathrm{p}<0.001$; median TTP: 8.4 vs 4.4 months). The ORR was $23 \%$ for the combination arm versus $14 \%$ for monotherapy $(p=0.113)$. Also, patients in lapatinib arm, experienced less central nervous system (CNS) metastases as compared to those treated with capecitabine alone (four cases vs 11 cases; $p=0.1$ ) [29]. An update of this trial confirmed the previously reported results [30]. Preclinical models demonstrated the interaction of lapatinib with trastuzumab as synergistic and resulting in enhanced apoptosis in HER2-positive BC cells [31]. In HER2positive xenograft models, lapatinib combined with trastuzumab resulted in stabilization of inactive HER2 receptor as a result of a mechanism of action attributed to lapatinib, followed by receptor degradation attributed to trastuzumab activity, with subsequent complete tumor regression within 10 days of combination treatment [32]. These preclinical data provide a rationale to pursue the combination of lapatinib and trastuzumab in the clinical setting. EGF104900 a phase III, randomized, multicenter, open-label study [33], was designed to compare the efficacy and safety of lapatinib alone with lapatinib in combination with trastuzumab in patients with HER2-positive MBC who had experienced progression on prior trastuzumab-based therapy. Patients were randomized to receive either oral lapatinib $1500 \mathrm{mg}$ daily or oral lapatinib $1000 \mathrm{mg}$ daily in combination with intravenous trastuzumab $2 \mathrm{mg} / \mathrm{kg}$ weekly (after the initial $4 \mathrm{mg} / \mathrm{kg}$ loading dose). The primary endpoint was progressionfree survival (PFS). The secondary endpoints included overall tumor response rate (ORR; confirmed complete response $[\mathrm{CR}]$ plus partial response $[\mathrm{PR}])$, clinical benefit response rate $(\mathrm{CBR}$; confirmed $\mathrm{CR}$ plus $\mathrm{PR}$ at any 
time, plus stable disease for $\geq 24$ weeks), overall survival (OS), quality of life (QOL), and safety. The combination of lapatinib with trastuzumab provided a statistically significant improvement in PFS compared with single-agent lapatinib, with an HR of $0.73(95 \%$ CI, 0.57 to $0.93 ; \mathrm{p}=$ $0.008)$. The median PFS was 8.1 weeks with lapatinib alone compared with 12.0 weeks with the combination treatment. The percentage of patients whose disease was progression free at 6 months was doubled in the combination arm compared with the monotherapy arm (28\% vs $13 \%$, respectively; $p=0.003)$. The ORR $(10.3 \%$ with the combination therapy vs $6.9 \%$ with lapatinib monotherapy) was not significantly different between the treatment arms $(p=0.46)$. The clinical benefit response $(\mathrm{CBR})$ reached statistical significance between the treatment groups ( $p=0.01$ ) with $24.7 \%$ of patients in the combination arm experiencing clinical benefit compared with $12.4 \%$ in the monotherapy arm. The median OS time was 51.6 weeks in patients receiving lapatinib plus trastuzumab compared with 39 weeks in patients receiving lapatinib monotherapy. The overall incidence of adverse events (AEs) was similar between treatment arms. Most events resolved without the need for dose modification, and the majority of AEs were grade 1 or 2 in severity. The incidence of diarrhea was significantly higher with the combination therapy, likely attributable to overlapping toxicities, because diarrhea represents an AE frequently reported for lapatinib and commonly reported also for trastuzumab. Despite the association of cardiac dysfunction with individual use of lapatinib and trastuzumab, the incidence of LVEF decreases with the combination of these two agents did not result in rates higher than what has been previously reported for each agent [7, 34]. This study demonstrated that lapatinib, in combination with trastuzumab, offers a chemotherapy-free option that has an acceptable tolerability profile and, vs lapatinib alone, reduces the risk of disease progression by $27 \%(p=0.008)$. These results support the preclinical activity of combined HER2 blockade and show that the combination of lapatinib and trastuzumab improves the outcome for patients with trastuzumab-refractory, HER2positive MBC over what would be expected for each agent alone. Other interesting associations, in phase III trials, have been those between lapatinib and paclitaxel in HER2- negative or unknown MBC patients [35]. In the ITT analysis 579 patients were randomly assigned to first-line therapy with paclitaxel $175 \mathrm{mg} / \mathrm{m}^{2}$ every 3 weeks plus lapatinib $1500 \mathrm{mg} /$ day or placebo. There were no statistically significant differences in time to progression (TTP), event-free survival (EFS) and OS between treatment arms, although an advantage in ORR ( $35.1 \%$ vs $25.3 \%, \mathrm{p}=0.008)$ and CBR $(40.5 \%$ vs $31.9 \%$, $\mathrm{p}=0.025)$ was observed. A subset analysis performed on 86 HER2-positive MBC patients revealed the statistically significant superiority of lapatinib plus paclitaxel, compared with paclitaxel plus placebo, in terms of TTP (36.4 vs 25.1 weeks, HR $0.53 ; \mathrm{p}=0.005)$, EFS ( 35.1 vs 21.9 weeks, HR $0.52 ; \mathrm{p}=0.004)$, ORR $(63.3 \%$ vs $37.8 \% ; \mathrm{p}=$ $0.023)$ and CBR $(69.4 \%$ vs $40.5 \% ; p=0.011)$. No differences in cardiac events were seen.

Very recently the preliminary results of a trial called EMILIA were presented [36]. This trial compared the combination of lapatinib/capecitabine to TDM1 in patients previously treated with trastuzumab. T-DM1 is a three-part of immuno-conjugate consisting of trastuzumab, a stable linker, and the potent emtansine derivative, DM1. This new compound incorporates the antitumor activity of trastuzumab with its ability to deliver a microtubule disrupting cytotoxic agent specifically to antigen-expressing tumor cells. Patients, previously treated with a taxane and trastuzumab, were eligible for the study if they had progressive disease during treatment for metastases or had recurrent disease within 6 months of adjuvant therapy. Stratification factors included world region, number of prior chemotherapy regimens and presence of visceral disease. Although 991 patients were enrolled, 978 patients were treated in the study. Median dose intensity was $99.9 \%$ for those receiving T-DM1, $77.2 \%$ for those receiving capecitabine and $93.4 \%$ for those treated with lapatinib. Dose reduction was necessary for only $16.3 \%$ of patients in the T-DM1 arm; however, the capecitabine dose and the lapatinib dose had to be reduced for $53.4 \%$ and $27.3 \%$ of patients, respectively. Median PFS was 9.6 months in the T-DM1 arm compared with 6.4 months in the capecitabine/lapatinib arm (stratified hazard ratio $[\mathrm{HR}]=0.650,95 \%$ CI: $0.55-0.77$; $\mathrm{p}<0.0001)$. Subgroup analyses according to baseline characteristics indicated that T-DM1 was better for all groups except for those patients ages 65 and older. OS was improved for patients receiving T-DM1, but median OS has not been reached for these patients and the efficacy boundary has not been crossed. The objective response rate was significantly higher in the T-DM1 group at $43.6 \%$ compared with $30.8 \%$ in the capecitabine/lapatinib group (95\% CI: $6.0-19.4, \mathrm{p}=0.0002)$. T-DM1 was much safer than capecitabine/lapatinib. Median time to symptom progression was 7.1 months in the T-DM1 group and 4.6 months in the capecitabine/lapatinib group $(\mathrm{HR}=0.80,95 \% \mathrm{CI}: 0.67-0.95, \mathrm{p}=0.0121)$. The incidence of grade 3 or higher severe adverse events was lower in the T-DM1 group (40.8\%) than in the capecitabine/lapatinib group $(57.0 \%)$, as was the incidence of adverse events resulting in treatment discontinuation (5.9\% vs $10.7 \%$, respectively). Cardiac toxicity was not increased. There was one death due to toxicity in the TDM1 group and five in the capecitabine/lapatinib group.

The Ma.31 study explored the relative efficacy of lapatinib vs trastuzumab when combined with taxane che- 
motherapy in the first-line setting of metastatic breast cancer. In the recently reported interim analysis, $636 \mathrm{pts}$ (525 HER2 centrally confirmed) were included [37]. In the intention to treat analysis, PFS was inferior with lapatinib compared to trastuzumab [hazard ratio $(\mathrm{HR})=$ $1.33 ; 95 \%$ CI $1.06-1.67 ; \mathrm{p}=0.01]$. In fact, lapatinib treated patients had a median PFS of 8.8 months compared to trastuzumab of 11.4 months, respectively. No difference in overall survival was detected, but more grade 3 - 4 diarrhea and rash was observed with the combination of lapatinib/taxane $(\mathrm{p}<0.001)$.

\section{Lapatinib in Brain Metastases from Breast Cancer}

In recent years brain metastases (BM) from breast carcinoma have become an increasing problem associated with a detrimental impact on survival and quality of life. In fact, BM are present in approximately $10 \%-16 \%$ of patients with metastatic breast disease [38].

The incidence of CNS metastases seems to have increased in recent years, probably owing to the prolonged survival of patients who underwent aggressive therapy for primary tumors, as well as improved technologies for detecting subclinical disease. For patients with BM occurring in a functional area or too large, numerous, or disseminated for surgery or radio-surgery, whole-brain radiotherapy (WBRT) remains the standard treatment, providing control of symptoms and increasing overall survival [39]. Little is known about predictive risk factors that allow the identification of breast cancer patients at risk of CNS metastases. The association of CNS metastases with HER2 overexpression merits a special mention. The amplification of the HER2 gene is correlated with diminished disease-free and overall survival, and is the strongest predictor of first relapse in brain site. Although trastuzumab reduces the risk of distant relapse, the CNS remains a site of initial and subsequent relapse. Several trials, have reported a high incidence of CNS metastases among patients treated with trastuzumab for stage IV breast cancer, approximately ranging from 28 to $43 \%$ [40]. These and other data suggest that the bloodbrain barrier (BBB) restricts the entry of large molecules into the brain, preventing trastuzumab from reaching adequate concentrations in the CNS [41]. Therefore, clinical trials have been carried out with lapatinib for the treatment of BM because it is a small molecule able to penetrate the blood-brain barrier. In the study of Geyer et al. [29] the patients in lapatinib arm, experienced less CNS metastases as compared to those treated with capecitabine alone (4 vs 11 cases, $p=0.1$ ). An update of this trial confirmed that the difference in the progression to CNS has become statistically significant (4 vs 13 cases, $p$ $=0.045$ ) [30]. In a phase II trial, Lin et al. explored the role of lapatinib in new or progressive BM from breast cancers overexpressing HER2. All 39 patients developed BM during treatment with trastuzumab and 38 progressed after prior radiation therapy. Two patients $(5 \%)$ achieved a partial response (PR) and remained on study for 158 and 347 days, respectively. Eight patients had steady disease in the CNS at 16 weeks [42]. The volumetric decline in CNS lesions correlated with improvements of quality of life. Recently, in a multicenter phase II trial, that enrolled more than 240 patients with progressive HER2+ brain metastases after cranial radiotherapy and trastuzumab, it was observed 6\% CNS objective response rate to lapatinib. In an exploratory analysis, $21 \%$ of patients achieved at least a $20 \%$ reduction in the volume of their CNS lesions. Additional responses were documented in patients who joined an optional extension phase of the study and who were treated with lapatinib plus capecitabine after progression on lapatinib alone [43]. On the basis of the results of this study, prospective studies of lapatinib in combination with other chemotherapeutic agents, novel targeted agents and cranial radiotherapy are ongoing.

\section{Conclusion}

In conclusion, lapatinib is an active and well tolerated oral dual TK inhibitor for the treatment of breast cancer. Clinical efficacy of lapatinib is limited only to the treatment of ErbB-2 overexpressing breast cancer. Lapatinib is active in refractory metastatic breast cancer patients and as a first-line metastatic treatment, with potential benefit in patients with brain metastases. Lapatinib has demonstrated efficacy in combination with capecitabine in patients with refractory ErbB-2 overexpressing breast cancer and in combination with letrozole in first line treatment of ER/Her2 positive patients. Moreover, the combination with trastuzumab is very promising in heavily pretreated patients. Lapatinib appears to have either very low or no incidence of cardiac toxicity. The most frequently reported adverse events include diarrhea, nausea, fatigue, itching, rash, acne, and dry skin. However, grade $3-4$ toxicities are rare, and most adverse events associated with lapatinib are of grade 1 o 2 . Our improved understanding of the biology of breast cancer and the use of biomarkers for the identification of specific subtypes of breast cancer allows us to bring patient specific novel therapies such as lapatinib to the clinic. Results from the phase III trials with lapatinib in combination regimens and from adjuvant and neo-adjuvant trials are eagerly awaited.

\section{REFERENCES}

[1] M. Press, L. Bernstein, P. Thomas, et al., "HER-2/neu Gene Amplification Characterized by Fluorescence in 
Situ Hybridization: Poor Prognosis in Node-Negative Breast Carcinomas," Journal of Clinical Oncology, Vol. 15, No. 8, 1997, pp. 2894-2904.

[2] G. Von Minckwitz, A. du Bois, M. Schmidt, et al., "Trastuzumab beyond Progression in Human Epidermal Growth Factor Receptor 2-Positive Advanced Breast Cancer: A German Breast Group/Breast International Group 03-05 Study," Journal of Clinical Oncology, Vol. 27, 2009, pp. 1999-2006. doi:10.1200/JCO.2008.19.6618

[3] M. Giampaglia, V. E. Chiuri, et al., "Lapatinib in Breast Cancer: Clinical Experiences and Future Perspectives," Cancer Treatment Reviews, Vol. 36, No. S3, 2010, pp. S72-S79. doi:10.1016/S0305-7372(10)70024-4

[4] W. Xia, L. H. Liu, P. Ho and N. L. Spector, "Truncated ErbB2 Receptor (p95ErbB2) Is Regulated by Heregulin through Heterodimer Formation with ErbB3 Yet Remains Sensitive to the Dual EGFR/ErbB2 Kinase Inhibitor GW572016," Oncogene, Vol. 23, No. 3, 2004, pp. 646653.

[5] R. Nahta, L. X. Yuan, Y. Du, et al., "Lapatinib Induces Apoptosis in Trastuzumab-Resistant Breast Cancer Cells: Effects on Insulin-Like Growth Factor 1 Signaling," Molecular Cancer Therapeutics, Vol. 6, 2007, pp. 667-674. doi:10.1158/1535-7163.MCT-06-0423

[6] A. Mukherjee, A. S. Dhadda, M. Shehata, et al., "Lapatinib: A Tyrosine Kinase Inhibitor with a Clinical Role in Breast Cancer," Expert Opinion on Pharmacotherapy, Vol. 8, No. 13, 2007, pp. 2189-2204.

[7] E. A. Perez, M. Koehler, J. Byrne, et al., "Cardiac Safety of Lapatinib: Pooled Analysis of 3689 Patients Enrolled in Clinical Trials," Mayo Clinic Proceedings, Vol. 83, No. 6, 2008, pp. 679-686.

[8] G. Arpino, L. Weichmann, C. K. Osborne, et al., "Crosstalk between the Estrogen Receptor and the HER Tyrosine Kinase Receptor Family," Endocrine Reviews, Vol. 29, 2008, pp. 217-233. doi:10.1210/er.2006-0045

[9] A. Hurtado, K. A. Holmes, T. R. Geistlinger, et al., "Regulation of ERBB2 by Estrogen Receptor-PAX2 Determines Response to Tamoxifen," Nature, Vol. 456, 2008, pp. 663-666. doi:10.1038/nature07483

[10] Z. Yang, C. J. Barnes and R. Kumar, "Human Epidermal Growth Factor Receptor 2 Status Modulates Subcellular Localization of and Interaction with Estrogen Receptor Alpha in Breast Cancer Cells," Clinical Cancer Research, Vol. 10, No. 11, 2004, pp. 3621-3628.

[11] L. A. Martin, I. Farmer, S. R. Johnston, et al., "Enhanced Estrogen Receptor (ER) Alpha, ERBB2, and MAPK Signal Transduction Pathways Operate during the Adaptation of MCF-7 Cells to Long Term Estrogen Deprivation," Journal of Biological Chemistry, Vol. 278, No. 33, 2003, pp. 30458-30468.

[12] S. Massarweh, C. K. Osborne, S. Jiang, et al., "Mechanisms of Tumor Regression and Resistance to Estrogen Deprivation and Fulvestrant in a Model of Estrogen Receptor-Positive, HER-2/neu-Positive Breast Cancer," Cancer Research, Vol. 66, No. 16, 2006, pp. 8266-8273.

[13] J. Shou, S. Massarweh, C. Osborne, et al., "Mechanisms of Tamoxifen Resistance: Increased Estrogen ReceptorHER2/neu Cross-Talk in ER/HER2-Positive Breast Can- cer," Journal of the National Cancer Institute, Vol. 96, No. 12, 2004, pp. 926-935.

[14] J. R. Mackey, B. Kaufman, M. Clemens, et al., "Trastuzumab Prolongs Progression-Free Survival in HormoneDependent and HER2-Positive Metastatic Breast Cancer," Breast Cancer Research and Treatment, Vol. 100, No. S5, 2006, Abstr 3.

[15] S. Johnston, J. Pippen Jr., et al., "Lapatinib Combined with Letrozole versus Letrozole and Placebo as First-Line Therapy for Postmenopausal Hormone Receptor-Positive Metastatic Breast Cancer," Journal of Clinical Oncology, Vol. 27, No. 33, 2009, pp. 5538-5546. doi:10.1200/JCO.2009.23.3734

[16] J. Lemieux, M. Clemons, L. Provencher, et al., "The Role of Neoadjuvant HER2-Targeted Therapies in HER2Overexpressing Breast Cancers," Current Oncology, Vol. 16, No. 4, 2009, pp. 48-57.

[17] A. U. Buzdar, N. K. Ibrahim, D. Francis, et al., "Significantly Higher Pathologic Complete Remission Rate after Neoadjuvant Therapy with Trastuzumab, Paclitaxel, and Epirubicin Chemotherapy: Results of a Randomized Trial in Human Epidermal Growth Factor Receptor 2-Positive Operable Breast Cancer," Journal of Clinical Oncology, Vol. 23, No. 16, 2005, pp. 3676-3685.

[18] B. P. Coudert, L. Arnould, L. Moreau, et al., "Preoperative Systemic (Neo-Adjuvant) Therapy with Trastuzumab and Docetaxel for HER2-Overexpressing Stage II or III Breast Cancer: Results of a Multicenter Phase II Trial," Annals of Oncology, Vol. 17, 2006, pp. 409-414. doi:10.1093/annonc/mdj096

[19] Neo ALTTO (Neoadjuvant Lapatinib and/or Trastuzumab Treatment Optimisation) Study, 2010. http://clinicaltrials.gov/ct2/show/NCT00553358

[20] S.-D. Costa, C. Jackisch, et al., "Future Roles of Lapatinib in ErbB2-Positive Breast Cancer: Adjuvant and Neoadjuvant Trials," Breast Care, Vol. 5, Suppl. 1, 2010, pp. 2-24. doi: $10.1159 / 000285778$

[21] F. A. Holmes, Y. M. Nagarwala, V. A. Espina, L. A. Liotta, M. A. Danso, et al., "Correlation of Molecular Effects and Pathologic Complete Response to Preoperative Lapatinib and Trastuzumab, Separately and Combined Prior to Neoadjuvant Breast Cancer Chemotherapy [Abstract]," Journal of Clinical Oncology, Vol. 29, No. 15S, 2011, p. 506.

[22] V. Guarneri, A. Frassoldati, A. Bottini, et al., "Final Results of a Phase II Randomized Trial of Neoadjuvant Anthracycline-Taxane Chemotherapy plus Lapatinib, Trastuzumab, or both in HER2-Positive Breast Cancer (CHER-LOB Trial)," ASCO Annual Meeting 2011, Abstract No. 507.

[23] J. C. N. Chang, I. A. Mayer, A. Forero-Torres, et al. "TBCRC 006: A Multicenter Phase II Study of Neoadjuvant Lapatinib and Trastuzumab in Patients with HER2Overexpressing Breast Cancer," ASCO Annual Meeting 2011, Abstract No. 505.

[24] A. Robidoux, G. Tang and P. Rastogi, "Evaluation of Lapatinib as a Component of Neoadjuvant Therapy for HER2+ Operable Breast Cancer: NSABP Protocol B-41," Journal of Clinical Oncology, Vol. 30, 2012, Abstr. 
LBA506.

[25] H. Burstein, A. M. Storniolo, S. Franco, et al., "A Phase II, Open-Label, Multi-Center Study of Lapatinib in Two Cohorts of Patients with Advanced or Metastatic Breast Cancer Who Have Progressed While Receiving Trastuzumab-Containing Regimens," Annals of Oncology, Vol. 15, Suppl 3, 2004, p. 27.

[26] N. U. Lin, V. Dieras, D. Paul, et al., "A Phase II Study of Lapatinib for Brain Metastases in Subjects with ErbB2Positive Breast Cancer Following Trastuzumab-Based Systemic Therapy and Cranial Radiotherapy," Proceedings of American Society of Clinical Oncology, Vol. 27, 2007, Abstr 1012.

[27] ALTTO (Adjuvant Lapatinib and/or Trastuzumab Treatment Optimisation) Study, 2010.

http://clinicaltrials.gov/ct2/show/NCT00490139

[28] Tykerb Evaluation after Chemotherapy (TEACH), "Lapatinib versus Placebo in Women with Early-Stage Breast Cancer," 2010.

http://clinicaltrials.gov/ct2/show/NCT00374322

[29] C. E. Geyer, J. Forster, D. Lindquist, et al., "Lapatinib plus Capecitabine for HER2-Positive Advanced Breast Cancer," New England Journal of Medicine, Vol. 355, No. 26, 2006, pp. 2733-2743.

[30] D. Cameron, M. Casey, M. Press, et al., "A Phase III Randomized Comparison of Lapatinib plus Capecitabine versus Capecitabine Alone in Women with Advanced Breast Cancer That Has Progressed on Trastuzumab: Updated Efficacy and Biomarker Analyses," Breast Cancer Research and Treatment, Vol. 112, No. 3, 2008, pp. 533543.

[31] G. E. Konecny, M. D. Pegram, N. Venkatesan, et al., "Activity of the Dual Kinase Inhibitor Lapatinib (GW572016) against HER-2-Overexpressing and Trastuzumab-Treated BC Cells," Cancer Research, Vol. 66, 2006, pp. 1630-1639. doi:10.1158/0008-5472.CAN-05-1182

[32] M. Scaltriti, C. Verma, M. Guzman, et al., "Lapatinib, a HER2 Tyrosine Kinase Inhibitor, Induces Stabilization and Accumulation of HER2 and Potentiates Trastuzumab-Dependent Cell Cytotoxicity," Oncogene, Vol. 28, No. 6, 2009, pp. 803-814.

[33] K. L. Blackwell, H. J. Burstein, A. M. Storniolo, et al., "Randomized Study of Lapatinib Alone or in Combination with Trastuzumab in Women with ErbB2-Positive, Trastuzumab-Refractory Metastatic Breast Cancer," Journal of Clinical Oncology, Vol. 28, No. 7, 2010, pp. 11241308. doi:10.1200/JCO.2008.21.4437
[34] K. L. Smith, C. Dang and A. Seidman, "Cardiac Dysfunction Associated with Trastuzumab," Expert Opinion on Drug Safety, Vol. 5, No. 5, 2006, pp. 619-629.

[35] A. Di Leo, H. L. Gomez, Z. Aziz, et al., "Phase III, Double-Blind, Randomized Study Comparing Lapatinib plus Paclitaxel with Placebo plus Paclitaxel as First-Line Treatment for Metastatic Breast Cancer," Journal of Clinical Oncology, Vol. 26, No. 34, 2008, pp. 5544-5552.

[36] K. L. Blackwell, D. Miles, L. Gianni et al., "Primary Results from EMILIA, a Phase III Study of Trastuzumab Emtansine (T-DM1) versus Capecitabine (X) and Lapatinib (L) in HER2-Positive Locally Advanced Ormetastatic Breast Cancer (MBC) Previously Treated with Trastuzumab (T) and a Taxane," Journal of Clinical Oncology, Vol. 30, 2012, Abstr LBA1.

[37] K. A. Gelmon, F. Boyle, B. Kaufman et al., "Open-Label Phase III Randomized Controlled Trial Comparing Taxane-Based Chemotherapy (Tax) with Lapatinib (L) or Trastuzumab ( $\mathrm{T})$ as First-Line Therapy for Women with HER2+ Metastatic Breast Cancer: Interim Analysis (IA) of NCIC CTG MA.31/GSK EGF 108919," Journal of Clinical Oncology, Vol. 30, 2012, Abstr LBA671.

[38] N. U. Lin, J. R. Bellon and E. P. Winer, "CNS Metastases in Breast Cancer," Journal of Clinical Oncology, Vol. 22, 2004, pp. 3608-3617. doi:10.1200/JCO.2004.01.175

[39] D. Khuntia, P. Brown, J. Li and M. P. Mehta, "WholeBrain Radiotherapy in the Management of Brain Metastasis," Journal of Clinical Oncology, Vol. 24, No. 8, 2006, pp. 1295-1304. doi:10.1200/JCO.2005.04.6185

[40] A. J. Clayton, S. Danson, S. Jolly, et al., "Incidence of Cerebral Metastases in Patients Treated with Trastuzumab for Metastatic Breast Cancer," British Journal of Cancer, Vol. 91, No. 4, 2004, pp. 639-643.

[41] J. Stemmler, M. Schmitt, A. Willems, et al., "Brain Metastases in HER2-Overexpressing Metastatic Breast Cancer: Comparative Analysis of Trastuzumab Levels in Serum and Cerebrospinal Fluid," Journal of Clinical Oncology, Vol. 24, Suppl. 18, 2006.

[42] N. U. Lin, L. A. Carey, M. C. Liu, et al., "Phase II Trial of Lapatinib for Brain Metastases in Patients with Human Epidermal Growth Factor Receptor 2-Positive Breast Cancer," Journal of Clinical Oncology, Vol. 26, No. 12, 2008, pp. 1993-1999.

[43] N. U. Lin, V. Diéras, D. Paul, et al., "Multicenter Phase II Study of Lapatinib in Patients with Brain Metastases from HER2-Positive Breast Cancer," Clinical Cancer Research, Vol. 15, No. 4, 2009, pp. 1452-1459. doi:10.1158/1078-0432.CCR-08-1080 